\title{
Structural superlubricity of platinum on graphite under ambient conditions: The effects of chemistry and geometry
}

\author{
Alper Özoğul, ${ }^{1}$ Semran İpek,${ }^{2}$ Engin Durgun, ${ }^{2}$ and Mehmet Z. Baykara ${ }^{1,2,3, a)}$ \\ ${ }^{1}$ Department of Mechanical Engineering, Bilkent University, Ankara 06800, Turkey \\ ${ }^{2}$ UNAM-Institute of Materials Science and Nanotechnology, Bilkent University, Ankara 06800, Turkey \\ ${ }^{3}$ Department of Physics, Harvard University, Cambridge, Massachusetts 02138, USA
}

(Received 6 October 2017; accepted 7 November 2017; published online 21 November 2017)

\begin{abstract}
An investigation of the frictional behavior of platinum nanoparticles laterally manipulated on graphite has been conducted to answer the question of whether the recent observation of structural superlubricity under ambient conditions [E. Cihan, S. İpek, E. Durgun, and M. Z. Baykara, Nat. Commun. 7, 12055 (2016)] is exclusively limited to the gold-graphite interface. Platinum nanoparticles have been prepared by e-beam evaporation of a thin film of platinum on graphite, followed by post-deposition annealing. Morphological and structural characterization of the nanoparticles has been performed via scanning electron microscopy and transmission electron microscopy, revealing a crystalline structure with no evidence of oxidation under ambient conditions. Lateral manipulation experiments have been performed via atomic force microscopy under ambient conditions, whereby results indicate the occurrence of structural superlubricity at mesoscopic interfaces of $4000-75000 \mathrm{~nm}^{2}$, with a noticeably higher magnitude of friction forces when compared with gold nanoparticles of similar contact areas situated on graphite. Ab initio simulations of sliding involving platinum and gold slabs on graphite confirm the experimental observations, whereby the higher magnitude of friction forces is attributed to stronger energy barriers encountered by platinum atoms sliding on graphite, when compared with gold. On the other hand, as predicted by theory, the scaling power between friction force and contact size is found to be independent of the chemical identity of the sliding atoms, but to be determined by the geometric qualities of the interface, as characterized by an average "sharpness score" assigned to the nanoparticles. Published by AIP Publishing.

https://doi.org/10.1063/1.5008529
\end{abstract}

In classical mechanics research, the subject of friction has drawn considerable attention. While the fundamental mechanisms that govern frictional behavior at interfaces are still to be comprehensively understood, a major part of the interest in the subject arises from its implications in the fields of energy and consequently environment. ${ }^{1}$ Within this context, developments in our understanding of friction and its controlled mitigation at relevant interfaces and time as well as length scales are expected to yield significant returns in the form of energy savings.

Based on the perspective outlined above, efforts aimed at measuring and controlling friction have accelerated in the past few decades with the emergence of advanced computational methods and experimental tools. ${ }^{2}$ While fluid-based lubricants are typically used at length scales associated with conventional engineering applications to reduce friction, solid lubricants based on two-dimensional materials have emerged as potential alternatives for mechanical systems on micro- and nanometer length scales. ${ }^{3}$ On the other hand, the idea of structural superlubricity (alternatively referred to as structural lubricity or simply superlubricity) presents an intriguing alternative to the use of lubricants with the aim of reducing friction at interfaces formed by solid surfaces. ${ }^{4-11}$ In particular, structural superlubricity is based on the proposition that friction should diminish at an interface formed by two atomically flat and molecularly clean crystalline surfaces

${ }^{a)}$ E-mail: mehmet.baykara@bilkent.edu.tr with different lattice parameters and/or incommensurate orientation, due to atomic-scale structural mismatch.

While the principle of structural superlubricity is simple enough that its application seems rather straightforward at first glance, two major hurdles exist for the realization of the idea. First, structurally superlubric behavior is expected to break down as the size of the contact between the slider and the substrate gets larger, due to structurally commensurate regions forming at the interface as a result of atomic-scale deformations, a phenomenon that has been predicted via theory and simulations ${ }^{12-14}$ and recently confirmed by experiments. ${ }^{15}$ Second, the occurrence of structural superlubricity is thought to strictly require the absence of mobile thirdbodies (i.e., dirt molecules) at the interface formed by the atomically flat surfaces as these molecules would position themselves at potential energy minima and effectively lock the surfaces together, leading to the disappearance of structurally superlubric behavior, which is characterized by a sublinear dependence of the friction force on the contact area. ${ }^{16}$

Despite the fact that the realization of structural superlubricity under ambient conditions was not deemed possible for a long time due to the requirement of molecular cleanliness described above, recent experiments have shown that it is indeed possible to achieve structurally superlubric sliding under ambient conditions for a material system consisting of gold nanoparticles on graphite obtained via a combination of thermal deposition and post-deposition annealing. ${ }^{11}$ The key to this achievement has been shown to be the inert character 
of gold, namely, that it does not oxidize under ambient conditions and thus maintains an atomically flat interface with graphite, preventing penetration by dirt molecules. On the other hand, the question that needs to be answered to extend the potential applicability of the method is whether structural superlubricity is specific to the gold-graphite interface or whether it can be extended to other noble metals. Moreover, the theory of structural superlubricity specifically states that the scaling factor dictating the dependence of friction force on the contact area depends exclusively on the geometry of the interface (specifically, its shape, and the relative orientation between the slider and the substrate) and not the chemical identity of the atoms forming the interface. ${ }^{17}$ To determine whether structural superlubricity under ambient conditions can be extended to noble metals other than gold and to test the proposition that the scaling of friction force with the contact area depends exclusively on the geometry of the interface and not its chemical identity, we have performed lateral manipulation experiments on platinum nanoparticles situated on graphite.

The material system has been obtained by e-beam deposition of small amounts $(\sim 1 \mathrm{~nm})$ of platinum on freshly cleaved graphite under high vacuum and subsequent annealing in a rapid thermal annealing (RTA) instrument at $1000^{\circ} \mathrm{C}$ for $30 \mathrm{~min}$, similar to what has been previously done for gold nanoparticles. ${ }^{18}$ The resulting sample features platinum nanoparticles of various sizes situated on graphite as confirmed by scanning electron microscopy [SEM, Fig. 1(a)]. Additionally, transmission electron microscopy (TEM) reveals mostly straight edges for the platinum nanoparticles, confirming their crystalline character [Fig. 1(b)]. Since it has recently been determined that the absence of an oxide layer on the sliding
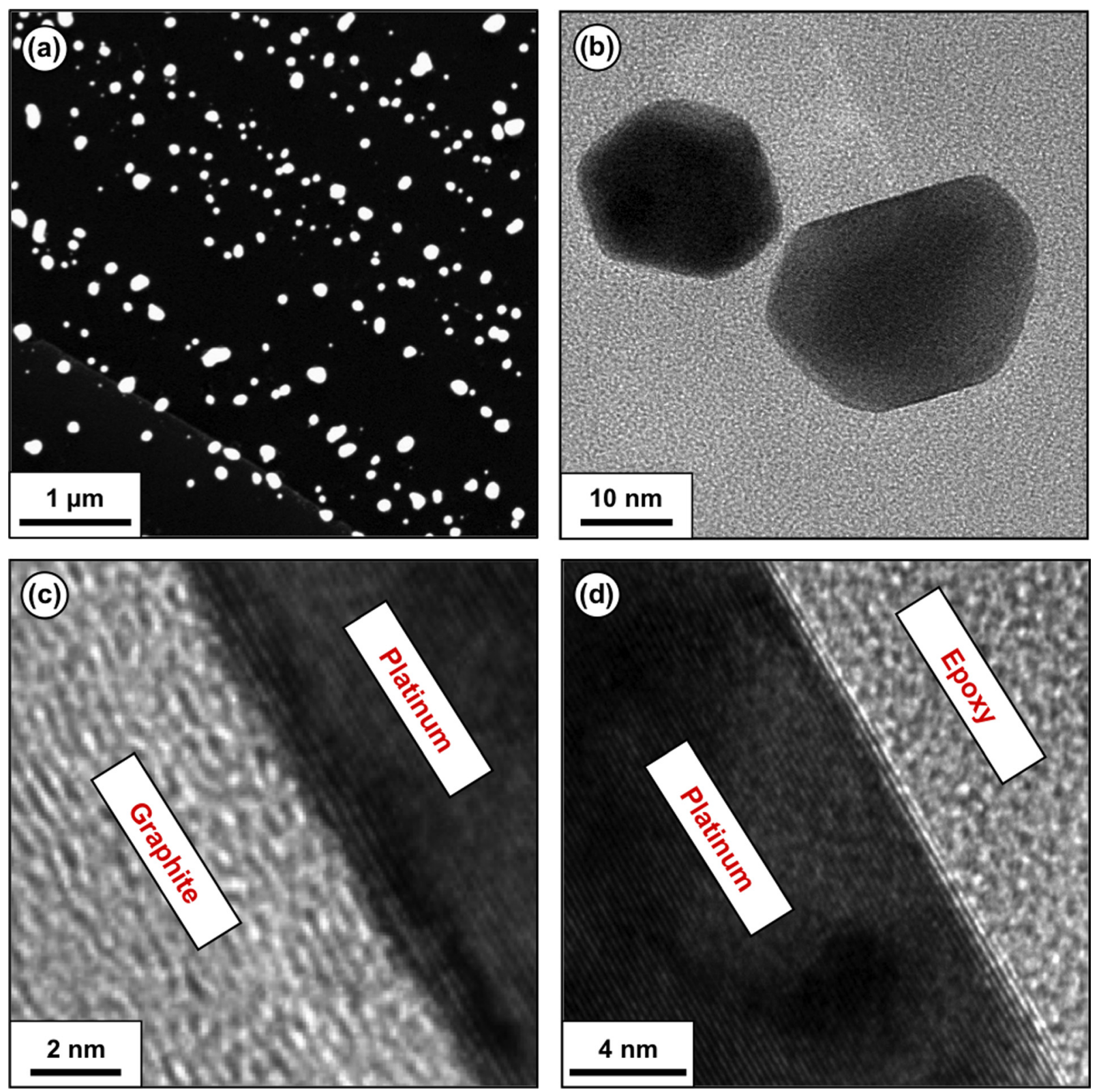

FIG. 1. (a) A representative SEM image of the investigated sample system consisting of platinum nanoparticles on graphite. (b) Top-down TEM image of two platinum nanoparticles exhibiting mainly straight edges indicative of crystallinity. (c) High-resolution cross-sectional TEM image (side view) of the bottom part of a platinum nanoparticle situated on graphite. (d) High-resolution cross-sectional TEM image (side view) of the top part of a platinum nanoparticle. Prior to TEM sample preparation via focused ion beam milling, the particle is covered by epoxy on its top side to prevent structural damage. ${ }^{11}$ 
surfaces is a prerequisite for structural superlubricity, crosssectional TEM measurements have been performed on the platinum nanoparticles, whereby the images in Figs. 1(c) and 1(d) show, in high resolution, the bottom and top sides of a particular platinum nanoparticle, respectively. The images confirm the crystalline character of the nanoparticle with (111) planes situated parallel to the graphite substrate, and the absence of an oxide layer, similar to the gold nanoparticles investigated previously. ${ }^{11}$

Lateral manipulation experiments have been performed on platinum nanoparticles of various sizes (4000$75000 \mathrm{~nm}^{2}$ ) using atomic force microscopy (AFM, PSIA $\mathrm{XE}-100 \mathrm{E})$ in the contact mode and commercial cantilevers (Nanosensors PPP-CONTR), using protocols described previously in detail. ${ }^{11,19}$ The normal and lateral calibrations of the cantilevers have been performed via well-established methods. ${ }^{20,21}$ The results of the manipulation experiments in terms of the friction forces $\left(F_{\mathrm{f}}\right)$ experienced by the particles as a function of contact area $(A)$ are reported in Fig. 2(a), both for the platinum particles investigated in this work, and for comparison purposes, for the gold nanoparticles that
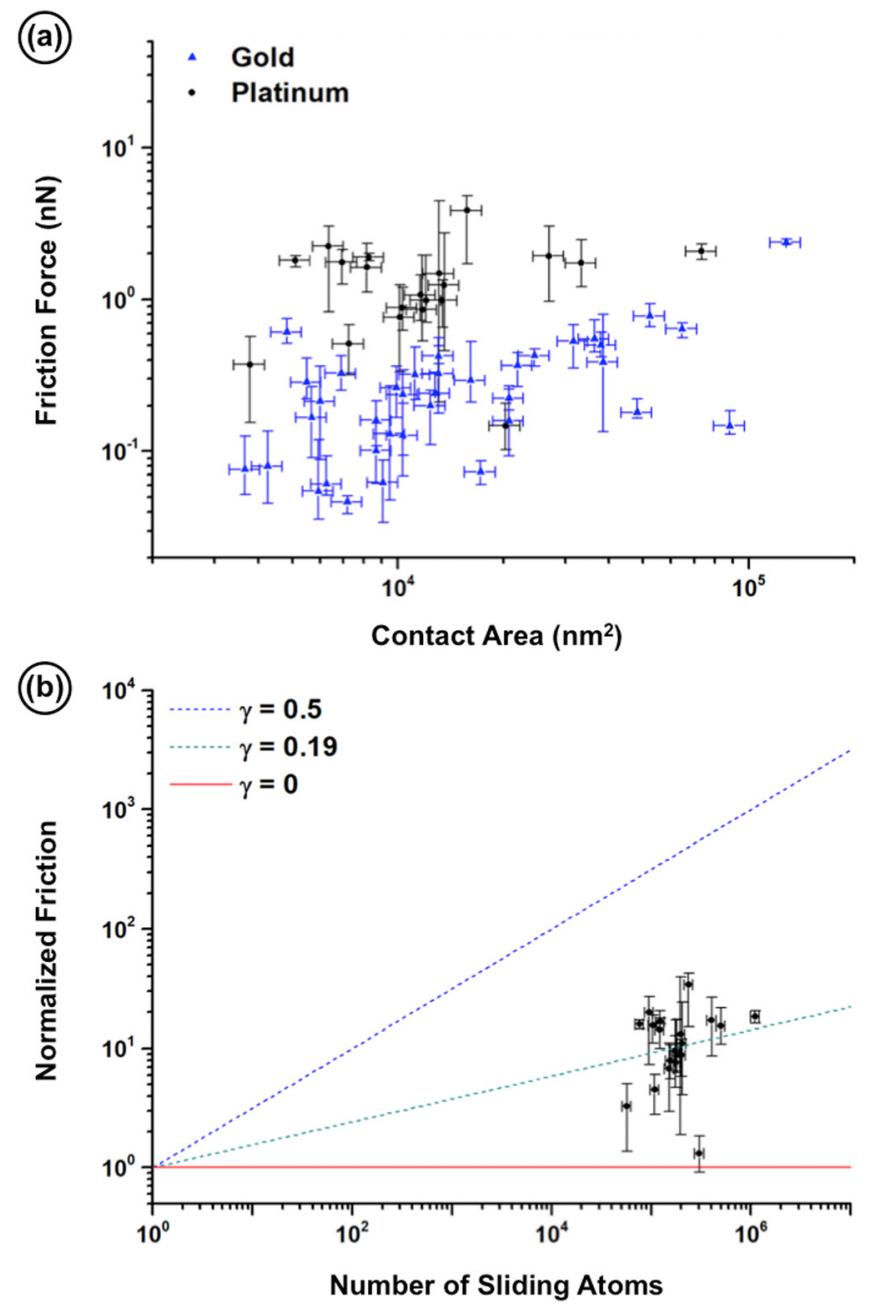

FIG. 2. (a) Friction force plotted as a function of contact area for platinum and gold nanoparticles. (b) Normalized friction $\left(F_{\mathrm{f}} / F_{0}\right)$ plotted as a function of number of sliding atoms, for platinum nanoparticles. Error bars related to friction are determined based on the maximum and minimum friction forces experienced by a given particle during the manipulation event. Error bars related to the contact area and number of sliding atoms are taken to be $\pm 10 \%$ due to tip-based convolution effects. ${ }^{29}$ have been measured previously. ${ }^{11}$ Remarkably, the friction forces measured for platinum particles are at least two orders of magnitude smaller than friction forces measured for antimony particles under ambient conditions using the same methodology, ${ }^{22}$ potentially indicative of structurally superlubric behavior. On the other hand, the measured friction forces for platinum nanoparticles are noticeably higher than those for gold nanoparticles, whereby the mean $F_{\mathrm{f}}$ values are $1.41 \mathrm{nN}$ and $0.33 \mathrm{nN}$, respectively.

Before the reasons behind the noticeable difference in friction force between platinum and gold are investigated, it needs to be determined whether the platinum nanoparticles indeed move in structurally superlubric fashion on graphite. The theory of structural superlubricity states that ${ }^{9}$

$$
F_{\mathrm{f}}=F_{0} N^{\gamma},
$$

where $F_{0}$ is the friction force expected for a single atom (determined as the ratio of the related diffusion barrier and the lattice constant), $N$ is the number of atoms on the sliding surface (determined by the multiplication of contact area values by the planar density of platinum atoms on (111) planes, which is 15 atoms $/ \mathrm{nm}^{2}$ ), and $\gamma$ is the scaling power which ranges from 0 to 0.5 depending on the shape of the slider and its commensurability with the underlying substrate. ${ }^{17}$ Using values of $173 \mathrm{meV}$ for the single-atom diffusion barrier ${ }^{23}$ and $0.246 \mathrm{~nm}$ for the lattice constant, the normalized friction $\left(F_{\mathrm{f}} / F_{0}\right)$ is plotted against $N$ on a log-log scale in Fig. 2(b) and it is confirmed that all manipulation events fall in the structural superlubricity regime $(0<\gamma<0.5)$, with an average $\gamma$ value of $0.19 \pm 0.06$.

While our measurements firmly confirm that structural superlubricity under ambient conditions is not exclusive to the gold-graphite interface and can also be observed on other noble metals such as platinum, it is also true that absolute friction force values measured for platinum are noticeably higher than those for gold. Considering that previous work has shown that platinum atoms interact in a significantly stronger way with graphene surfaces than gold (as characterized, e.g., by much larger adsorption energies), ${ }^{23}$ this result may not be surprising. On the other hand, to independently confirm the observation of higher friction forces for platinum nanoparticles sliding on graphite when compared with gold, we have performed ab initio simulations of 3-layer platinum and gold slabs sliding on 5-layer graphite substrates in structurally incommensurate configurations [Fig. 3(a)]. The simulations, based on density functional theory (DFT), have been performed using the Vienna $A b$ Initio Simulation package (VASP). ${ }^{24,25}$ The exchange-correlation has been approximated by the generalized gradient approximation (GGA) using the Perdew-Burke-Ernzerhof functional with van der Waals correction. ${ }^{26,27}$ The projector augmentedwave method (PAW) has been utilized for pseudopotentials of carbon, platinum, and gold with a plane-wave basis set having a kinetic energy cut-off of $400 \mathrm{eV} .^{28}$ The metal slabs have been slid on the graphite substrate along the $x$ direction [see Fig. 3(a)] in steps of $0.2 \AA$, and the resulting changes in the total energy of the system have been calculated at each step. After each step, the metal slabs were allowed to relax in the direction normal to the substrate surface. The energy 
(a))
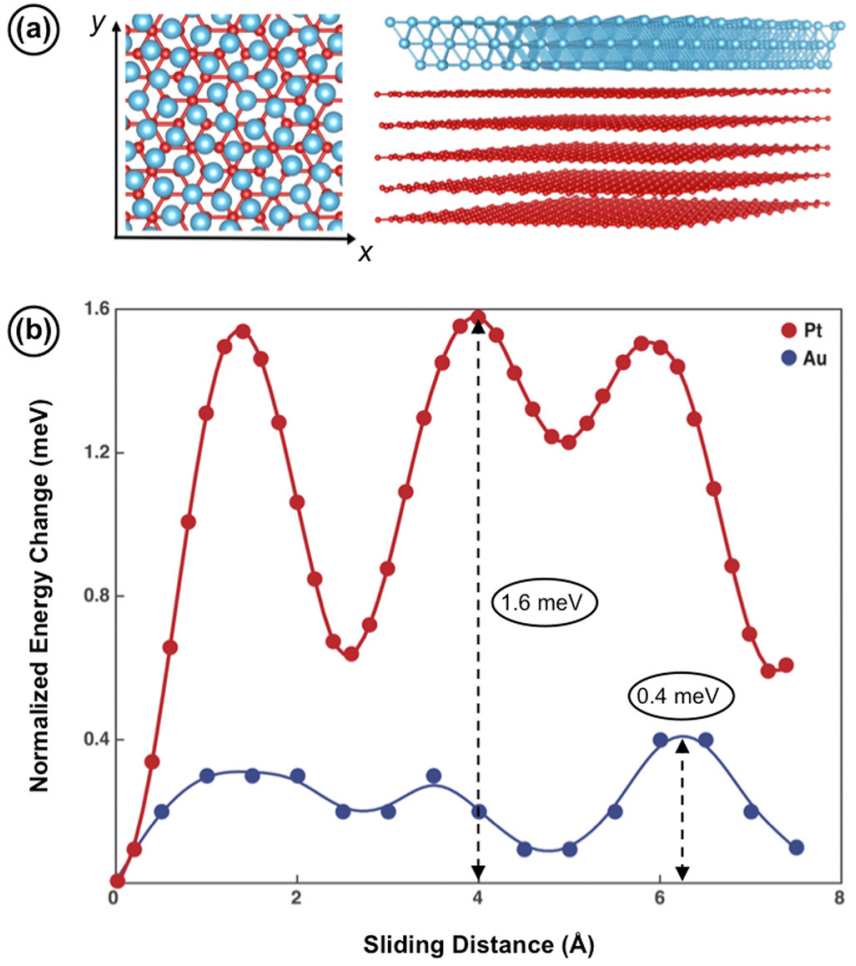

FIG. 3. (a) Top-down (left) and side (right) views of the model system used for the $a b$ initio simulations, consisting of a 3-layer metallic slab (blue atoms) and 5-layer graphite substrate (red atoms). While simulations are performed for both platinum and gold slabs, the platinum slab is depicted for visualization purposes. (b) Calculated change in the total energy of the system, normalized per sliding metal atom, as a function of sliding distance in the direction $x$. While the largest energy barrier encountered by platinum atoms is calculated as $1.6 \mathrm{meV}$, the corresponding value for gold atoms is $0.4 \mathrm{meV}$.

changes, normalized based on the number of atoms on the sliding metal surface, are plotted as a function of the sliding distance, for both platinum and gold slabs in Fig. 3(b). The results clearly show that platinum atoms experience significantly higher energy barriers than gold while laterally moving on the substrate $(1.6 \mathrm{meV}$ vs. $0.4 \mathrm{meV}$, considering the highest energy barriers in the direction of motion) and consequently larger resistance to motion in the lateral direction. Taking into account the similar planar densities of gold and platinum on (111) planes (14 atoms $/ \mathrm{nm}^{2}$ vs. 15 atoms/ $\mathrm{nm}^{2}$ ), this result suggests that for platinum and gold nanoparticles with similar contact areas (and in the absence of shape effects), the friction force is expected to be about four times larger in the case of platinum, which is closely reproduced in our experiments.

At this point, it needs to be noted that the friction force is not only a function of the slider-substrate interaction strength (as determined by $F_{0}$ ) but also of the scaling power $\gamma$. Assuming that the manipulated nanoparticles are not (or at least, do not remain) in pseudo-commensurate positions with the substrate, $\gamma$ is only determined by the geometry of the contact area, in particular, the sharpness of its circumference. In fact, it has been shown before via simple simulations based on analytic potentials that crystalline particles with perfectly round shapes are expected to feature a $\gamma$ of 0.25 while sliding on a crystalline substrate in incommensurate configuration, whereas particles with exclusively straight edges and sharp corners (such as a triangle) are expected to slide with a $\gamma$ of $0 .{ }^{9,17}$ Realistic contact geometries are between the two extremes and as such, $\gamma$ values between 0 and 0.25 are to be expected depending on the geometry of the contacts. To take the "sharpness effect" into account, an image analysis procedure has been conducted on SEM images of platinum and gold nanoparticles. SEM images have been processed by first converting the images into grayscale and then by the application of a threshold to the image to separate the nanoparticles from the background. Nanoparticles that coincide with the borders of the SEM images have been removed, and the rest of the nanoparticles have been filtered according to their sizes, since the sharpness calculation is prone to errors in smaller shapes. Accordingly, particles that cover less than $0.1 \%$ of a given image have been filtered out. Circumferences of each nanoparticle have been sliced into small segments, and the slopes of the lines that are tangent to each segment have been calculated. The differences of consecutive slopes have been recorded and a score of 0 has been assigned to vanishingly small differences, which suggest a straight line, and a score of 100 has been assigned to significant differences. Subsequently, the average "sharpness score" for each particle has been calculated, which is a measure of how straight or curved the circumference of a given particle is on average. In this calculation, a perfect circular or elliptical circumference would achieve a score of 100 and a perfectly sharp polygonal circumference would achieve a score of 0 . To correct the sharpness score for polygonal shapes, a maximum difference threshold is set to eliminate the effect of the corners. A total of 22 platinum and 50 gold nanoparticles have been taken into account in the calculations. The average sharpness score of gold nanoparticles has been determined as $76.7 \pm 9.2$, whereas the average sharpness score of platinum nanoparticles has been determined as $80.2 \pm 8.2$. The close sharpness scores for platinum and gold nanoparticles suggest that the average $\gamma$ values also need to be similar, with potentially a slightly larger value for the platinum nanoparticles due to their slightly less sharp character. This prediction is seen to be accurate as the $\gamma$ values are $0.19 \pm 0.06$ for the platinum particles investigated here and $0.16 \pm 0.06$ for the gold nanoparticles investigated previously. ${ }^{11}$

In this letter, we have shown via AFM-based lateral manipulation experiments that structural superlubricity under ambient conditions is not exclusive to the gold-graphite interface and that it can be achieved also using another noble metal, namely, platinum, albeit with a noticeably higher magnitude of friction forces. The difference between the friction forces measured for the two materials, thought to originate from a difference in interaction strength with the substrate, has been reproduced in ab initio simulations. Moreover, no significant difference in scaling factors relating friction forces to the contact area has been found between the two materials, as expected from the similar sharpness of contact area circumference exhibited by the two types of nanoparticles. Consequently, we have experimentally verified the theoretical expectation that the scaling power between contact size and friction force is independent of the chemical identity of the sliding atoms in structural 
superlubricity but rather depends on the geometric qualities of the interface.

M.Z.B. acknowledges financial support from the Marie Curie Actions of the European Commission's FP7 Program in the form of a Career Integration Grant (PCIG12-GA2012-333843). M.Z.B. and E.D. acknowledge financial support from the Outstanding Young Scientist Program of the Turkish Academy of Sciences (TÜBA-GEBIP).

${ }^{1}$ K. Holmberg and A. Erdemir, Friction 5(3), 263 (2017).

${ }^{2}$ J. Krim, Surf. Sci. 500, 741 (2002).

${ }^{3}$ J. C. Spear, B. W. Ewers, and J. D. Batteas, Nano Today 10, 301 (2015).

${ }^{4}$ M. Hirano and K. Shinjo, Phys. Rev. B 41(17), 11837 (1990).

${ }^{5}$ K. Shinjo and M. Hirano, Surf. Sci. 283(1-3), 473 (1993).

${ }^{6}$ M. H. Muser, L. Wenning, and M. O. Robbins, Phys. Rev. Lett. 86, 1295 (2001).

${ }^{7}$ M. H. Muser, EPL 66, 97 (2004).

${ }^{8}$ M. Dienwiebel, G. S. Verhoeven, N. Pradeep, J. W. M. Frenken, J. A. Heimberg, and H. W. Zandbergen, Phys. Rev. Lett. 92, 126101 (2004).

${ }^{9}$ D. Dietzel, M. Feldmann, U. D. Schwarz, H. Fuchs, and A. Schirmeisen, Phys. Rev. Lett. 111, 235502 (2013).

${ }^{10}$ D. Berman, S. A. Deshmukh, S. K. R. S. Sankaranarayanan, A. Erdemir, and A. V. Sumant, Science 348, 1118 (2015).

${ }^{11}$ E. Cihan, S. Ipek, E. Durgun, and M. Z. Baykara, Nat. Commun. 7, 12055 (2016).
${ }^{12}$ M. Ma, A. Benassi, A. Vanossi, and M. Urbakh, Phys. Rev. Lett. 114, 055501 (2015).

${ }^{13}$ A. Benassi, M. Ma, M. Urbakh, and A. Vanossi, Sci. Rep. 5, 16134 (2015).

${ }^{14}$ T. A. Sharp, L. Pastewka, and M. O. Robbins, Phys. Rev. B 93, 121402 (2016).

${ }^{15}$ D. Dietzel, J. Brndiar, I. Stich, and A. Schirmeisen, ACS Nano 11, 7642 (2017).

${ }^{16}$ G. He, M. H. Muser, and M. O. Robbins, Science 284(5420), 1650 (1999).

${ }^{17}$ A. S. de Wijn, Phys. Rev. B 86, 085429 (2012).

${ }^{18}$ E. Cihan, A. Ozogul, and M. Z. Baykara, Appl. Surf. Sci. 354, 429 (2015).

${ }^{19}$ D. Dietzel, T. Monninghoff, L. Jansen, H. Fuchs, C. Ritter, U. D. Schwarz, and A. Schirmeisen, J. Appl. Phys. 102, 084306 (2007).

${ }^{20}$ J. E. Sader, J. W. M. Chon, and P. Mulvaney, Rev. Sci. Instrum. 70, 3967 (1999).

${ }^{21}$ M. Varenberg, I. Etsion, and G. Halperin, Rev. Sci. Instrum. 74, 3362 (2003).

${ }^{22}$ D. Dietzel, C. Ritter, T. Monninghoff, H. Fuchs, A. Schirmeisen, and U. D. Schwarz, Phys. Rev. Lett. 101, 125505 (2008).

${ }^{23}$ X. J. Liu, C. Z. Wang, M. Hupalo, W. C. Lu, M. C. Tringides, Y. X. Yao, and K. M. Ho, Phys. Chem. Chem. Phys. 14, 9157 (2012).

${ }^{24}$ G. Kresse and J. Furthmuller, Phys. Rev. B 54, 11169 (1996).

${ }^{25}$ G. Kresse and J. Furthmuller, Comput. Mater. Sci. 6, 15 (1996).

${ }^{26}$ J. P. Perdew, J. A. Chevary, S. H. Vosko, K. A. Jackson, M. R. Pederson, D. J. Singh, and C. Fiolhais, Phys. Rev. B 46, 6671 (1992).

${ }^{27}$ S. Grimme, J. Comput. Chem. 27, 1787 (2006).

${ }^{28}$ P. E. Blochl, Phys. Rev. B 50, 17953 (1994).

${ }^{29}$ C. Ritter, M. Heyde, B. Stegemann, K. Rademann, and U. D. Schwarz, Phys. Rev. B 71, 085405 (2005). 